\title{
Programa Cátedra universitaria para adultos mayores en el Tecnológico de Antioquia
}

\section{University chair program for the elderly at Antioquia the Tecnologico}

\author{
Nora Hincapié Gil*
}

\section{Resumen:}

El presente artículo da cuenta de la construcción del marco filosófico, metodológico y teórico del programa educativo de extensión académica "Cátedra universitaria para adultos mayores" en el Tecnológico de Antioquia; validado desde la experiencia directa con un grupo de cuarenta adultos mayores. Como proyecto piloto de implementación, inscrito en la línea de Equidad y desarrollo humano del grupo de investigación Senderos, de la Facultad de Educación.

Palabras clave: Adultos mayores; Cátedra; Universidad; Educación continua.

Gerontóloga, Especialista en Docencia Investigativa Universitaria, Tecnológico de Antioquia - Institución Universitaria nhincapi@hotmail.com 
Tea Nora Hincapié Gil

\begin{abstract}
:
This article reports the building of the philosophical, methodological and theoretical framework about the outreach educational program called "university chair for elderly people" at Antioquia the Tecnologico. This is as a pilot project of implementation suscribed inside the field of equality and human development of the research group Senderos of the Education Faculty of the same institution.
\end{abstract}

Keywords: Elderly; Professor; University; Continuing education.

\section{Horizonte filosófico del programa}

La cátedra universitaria para adultos mayores, es una oferta educativa de extensión académica delaFacultad de Educación y Ciencias Sociales que se inscribe en los procesos misionales universitarios en investigación, docencia y bienestar, como una propuesta de educación continua y proyección social.

Se proyecta en concordancia con los postulados propuestos por diferentes organismos internacionales y nacionales sobre educación para personas mayores; en la necesidad percibida en el medio de educación permanente, y con el deseo de adquirir nuevos conocimientos y actualizar otros; como respuesta a las demandas de un mundo cambiante y en constante avance tecnológico; y con los índices estadísticos de la población mundial, que evidencian el cambio en la pirámide poblacional, que aumenta cada vez más la población adulta mayor.

Este programa educativo fomenta el desarrollo en dimensiones individuales, familiares y sociales, desde la perspectiva del aprendizaje a lo largo de la vida para motivar, a personas que han acumulado experiencias laborales y profesionales y reflexionen sobre su propio quehacer permitiendo realizar actividades intelectuales y su participación en programas que incidan en el progreso de la sociedad.
Los programas universitarios para personas mayores, además del componente educativo, propician espacios intergeneracionales y de inclusión social, que fortalecen los asuntos académicos investigativos con el objeto de establecer políticas públicas en beneficio de este grupo poblacional.

El programa responde al objetivo: Ofrecer programas educativos, que integren las necesidades de capacitación de la población adulta mayor, fortaleciendo niveles intelectuales y psicosociales, en mejora de su calidad de vida.

Tiene como misión, "En la Universidad para Adultos Mayores, se capacitan personas comprometidas con su formación permanente, mediante programas de extensión académica universitaria, desde el modelo gerogógico, que permite potenciar procesos educativos, favoreciendo la inclusión social de este grupo poblacional”.

Propone como visión, "La Universidad para Adultos Mayores será identificada como un programa de educación permanente en el departamento de Antioquia y con proyección nacional, reconocida por el compromiso con el desarrollo humano y social, la calidad de vida de las personas mayores, respondiendo a los requerimientos educativos desde un enfoque de inclusión social”. 
Se propone como valores: respeto, compromiso, responsabilidad, autonomía, humanismo y equidad

\section{MARCO METODOLÓGICO DE LA CÁTEDRA UNIVERSITARIA PARA ADULTOS MAYORES}

La educación impone un sello fundamental en la vida del ser humano, así como hay una educación para los niños, que es la pedagogía infantil, hay una educación para los adultos, que es la andragogía y una educación para los adultos mayores, que es la gerogogía; como la ciencia y el arte de redescubrir todo el potencial humano en los adultos mayores, fruto de una serie de experiencias vividas a lo largo de su ciclo vital que capacita al ser humano como ser actuante de su propia educación.

La gerogogía como enfoque educativo para personas mayores permite una educación en todos los niveles; es decir, una educación no solamente para las poblaciones más recientes, sino para poblaciones con una cronología más avanzada, su interés está enfocado en la posibilidad de gestar y promover actividades educativas en poblaciones que salen del interés y focos de atención de la demanda en los modelos pedagógicos. La pretensión principal está fundamentada desde el fenómeno social que prevalece en los cambios científicos en las sociedades que envejecen, que vive la modernización y que afecta de manera especial la capacidad de desempeño y adaptación de los adultos mayores.

Esta práctica educativa posibilita seguir aprendiendo de acuerdo con las características específicas de cada adulto mayor, tanto de orden biológico, como psicosocial $y$, tiene como fin proporcionar elementos que aporten a una mejor calidad de vida. De acuerdo con las características que posee este grupo poblacional para desarrollar el proceso de aprendizaje, se puede dar mayor disposición cuando el tema se ajusta a sus roles y necesidades, pues cada persona aprende a su propio ritmo y solamente lo que le sirve y aprende con mayor facilidad, a través de la práctica. De este modo, aplica lo aprendido a las situaciones de la vida diaria, pues, en este caso, es la misma persona quien construye el conocimiento.

En el aprendizaje adquirido mediante estrategias gerogógicas, la persona mayor se responsabiliza de su propio aprendizaje mediante la práctica, lo que favorece el fortalecimiento de las habilidades $y$ competencias de las personas para su desempeño cotidiano. Adquiridas a partir de un referente activo y dinámico que le permite a cada persona acercarse a nuevos saberes a su propio ritmo y de acuerdo con intereses $\mathrm{y}$ necesidades que contribuyen al logro de cambios favorables en el comportamiento y actitudes.

La propuesta educativa está orientada por el método de la investigación cualitativa y la metodología de la investigación acciónparticipación, mediante un aprendizaje colectivo de la realidad, basado en un análisis con la participación de la población implicada, que se orienta a estimular la práctica transformadora y el cambio social. Se combinan las funciones de conocer y actuar y se involucra a la población cuya realidad se aborda. Esta metodología permite analizar variables administrativas, pedagógicas y de personas mayores dentro de un contexto universitario, con la indagación a expertos y 
Tdea Nora Hincapié Gil

población adulta mayor.

El área administrativa del programa educativo para adultos mayores, se ejecuta desde la planeación, programación de los cursos, consecución de docentes, divulgación de la información y las inscripciones de los estudiantes.

El modelo pedagógico está contextualizado con el modelo gerogógico a través de la revisión con profesionales en Gerontología y Educación que dé cuenta de la estrategia de enseñanza aprendizaje.

La Cátedra universitaria para adultos mayores está dirigida a personas mayores de 50 años, que deseen participar en procesos de enseñanza aprendizaje en entornos universitarios en los módulos de:

\section{Sistemas:}

Iniciación en sistemas, redes sociales virtuales. Este espacio permitirá el acceso de los participantes a recursos tecnológicos de actualidad y, por consiguiente, promoverá la participación e integración en grupos intergeneracionales y la inclusión en contextos que técnicamente permanecían aislados. El aspecto gerogógico fundamental es la indagación continua de conocimientos, en el que el instructor integra el desempeño y la realimentación para brindarles respuestas adecuadas a los interrogantes de los participantes.

\section{Literatura:}

Narración, interpretación y composición literaria. Tiene como objetivo la promoción y generación de actividades lecto- escriturales en los adultos mayores, como posibilidad de enriquecer su acontecer histórico. Se menciona en este caso lo histórico desde la perspectiva que cada ser humano porta, es decir, una historicidad que la ha venido constituyendo en su trayectoria. Si bien es importante rescatar la lectura como la posibilidad de enriquecer, desarrollar y potenciar la esfera de lo cognitivo, desde la propuesta del módulo, la pretensión es trascender la lectura y la escritura a un acto que no se debe entender como mecánico y superficial.

\section{Mantenimiento cognitivo:}

Gimnasia mental. Neuróbicos. El estado cognitivo del adulto mayor es imprescindible en la identificación de cambios tempranos en el estado fisiológico, la capacidad de aprendizaje y el declive del funcionamiento cognitivo y afectivo. En la actualidad, las generaciones de adultos mayores adquieren un mayor nivel académico, demandan programas que mantengan la mente activa. En el medio, se conocen con el nombre de Gimnasia mental, mantenimiento cognitivo, neuróbicos y neuroaeróbicos; todos apuntan a la terapia de la memoria. El módulo de Mantenimiento cognitivo se propone como una alternativa para que las personas mayores continúen en su proceso de enseñanza aprendizaje y ejerciten sus mentes y eviten el deterioro cognitivo e intelectual.

\section{Historia y cultura:}

Historia y patrimonio cultural de Medellín. Comprender parte de la historia y la cultura de Medellín, mediante las experiencias de vida con los estudiantes en distintos contextos de la ciudad, observar videos y leer textos que ilustren y evoquen lugares y momentos destacados de la historia y la cultura. Además, realizar visitas guiadas por algunos de los sitios históricos de Medellín (Palacio de la cultura, por ejemplo). 


\section{Gerontología:}

Envejecimiento

biopsicosocial. $\quad \mathrm{La}$ gerontología como disciplina que se ocupa del estudio del envejecimiento y la vejez, atiende los cambios progresivos biopsicosociales con el paso del tiempo. Pretende fortalecer los conocimientos y las implicaciones que trae este acontecimiento con el fin de intervenir preventivamente en procura de mejorar la calidad de vida de este grupo poblacional. Además, socializar los programas y servicios que existen en el medio para los adultos mayores, con el propósito de que tengan mayores posibilidades de un envejecimiento productivo.

\section{Gimnasia oriental:}

Yoga, taichí, pilates e hidroaeróbicos. El objetivo es ofrecer un programa de gimnasia oriental que propicie calidad de vida en las personas mayores, que tomen conciencia de la importancia de su propio cuerpo, a través de prácticas y ejercicios orientales ordenados, que beneficiarán su salud, su estado de ánimo y el mejoramiento de las relaciones interpersonales.

\section{FUNDAMENTACIÓN TEÓRICA DEL PROGRAMA UNIVERSITARIO PARA ADULTOS MAYORES}

La función mediadora del programa universitario para adultos mayores se ve reflejada en la gestión de posibilidades para una formación permanente, que se evidencia en el desarrollo de políticas de inclusión social y de la cultura que asume la población adulta mayor como una riqueza, que, a su vez, se constituye en una base sólida para lograr aprendizajes integrales que generen mayores niveles de satisfacción en la población.
Con base en lo anterior, la implementación de programas académicos universitarios para adultos mayores debe verse en términos de cambio e innovación; que permita establecer programas educativos de carácter gerogógico, dentro del entorno universitario en los programas de extensión y educación continua, que vislumbren programas de bienestar y proyección social, centrados en la inclusión de grupos intergeneracionales y que, además de estar inmerso en ambientes de enseñanza aprendizaje, permita el avance académico e investigativo.

Cuando se asume la educación para adultos mayores como enfoque gerogógico, la universidad adquiere un papel protagónico en la modificación de los valores, las actitudes y los prejuicios sociales actuales, respecto a las personas consideradas "de la tercera edad". De esta manera, genera presión en la sociedad para formular políticas sociales incluyentes y nuevos contextos para la convivencia.

El siguiente constructo da cuenta de los conceptos que se abordan para la referencia en el tema de educación para personas mayores.

\section{Antecedentes históricos de la educación gerontológica}

Así como las ciencias generan sus disciplinas, la gerontología ha demostrado que necesita nutrirse de otras disciplinas, y, en el enfoque de las prácticas pedagógicas con el adulto mayor, se ha definido con diferentes nombres como: Educación gerontológica, gerontología educativa, gerontagogía, geriagogía, geragogía y gerogogía.

Dado que la gerontología educativa propone que la persona mayor aprende de una manera 
TCleA Nora Hincapié Gil

distinta de los jóvenes, es necesario buscar métodos que estén orientados en función del aprendizaje específico del adulto mayor. El conjunto de métodos nuevos, técnicas, entre otros, agrupados en un nuevo corpus de conocimiento, originará el nacimiento de esta disciplina a la que se denomina como gerontagogía, difundida como una disciplina educativa interdisciplinar, que tiene por objeto de estudio la persona mayor en situación educativa. Para Lemieux (1997) "Etimológicamente proviene del griego "gerontagogeo", que significa conducir a un viejo”.

\section{Derechos humanos y políticas públicas de educación para personas mayores}

Una política pública orientada a un grupo específico como lo es el de las personas adultas mayores, reconoce la vulnerabilidad de los derechos de ese grupo poblacional, en este caso, las personas mayores. Los derechos humanos, dentro de sus objetivos fundamentales, expresan la responsabilidad compartida que debe existir entre los diferentes actores, como: la familia, las personas y los actores sociales. En especial el Estado, quien es directamente implicado, está interesado en que a la población no se le vulneren sus derechos; por ello, busca la asesoría, asistencia técnica territorial e institucional, capacitación y educación continua, desarrollo de capacidades individuales y colectivas para el ejercicio efectivo del derecho.

No se habla de la educación como un lujo al alcance de una minoría sino de un derecho fundamentado desde la misma declaración de principios universales; no obstante, a determinadas edades, no se puede pensar en beneficios previstos a largo plazo. Parecen evidentes las transformaciones acaecidas en los últimos años en la sociedad que no hubieran sido posibles de no ser por la ayuda y servicios, con frecuencia voluntarios, de las personas de más edad.

En el Plan de acción internacional sobre envejecimiento y vejez, Viena 1982, en recomendaciones para la acción en cuanto a educación expresa: Recomendación 47De conformidad con el concepto de la Organización de las Naciones Unidas para la Educación, la Ciencia y la Cultura (Unesco) sobre la educación permanente, se recomienda promover programas para personas con avanzada edad, no estructurados, basados en la comunidad y orientados al esparcimiento, con el fin de adoptar un sentido de autosuficiencia, así como la responsabilidad de la comunidad respecto a las personas de edad avanzada. Tales programas deben contar con el apoyo de los gobiernos nacionales y de las organizaciones internacionales, como lo plantea Naciones Unidas (1982).

El Plan de acción internacional sobre envejecimiento y vejez, Madrid 2002, en el tema de acceso al conocimiento, la educación y la capacitación, expresa: La educación es una base indispensable para una vida activa y plena. Una sociedad basada en el conocimiento requiere la adopción de políticas para garantizar el acceso a la educación y la capacitación durante todo el curso de la vida. La educación y la capacitación permanentes son indispensables para conseguir la productividad de los trabajadores y de las naciones. Los cambios tecnológicos pueden contribuir a la alienación de las personas de edad carentes de educación o capacitación. Por consiguiente, habría que adoptar medidas 
para permitir el acceso, la participación y la adaptación a los cambios tecnológicos de las personas de edad, recomendación de Naciones Unidas (2002).

En Colombia, la Ley 1251 del 27 de noviembre de 2008, dicta normas de los derechos de los adultos mayores, en cuanto a educación se refiere, propone programas educativos para las personas mayores que les permitan la participación ciudadana $\mathrm{y}$ la equidad en todos los campos de interés, propicia un mejoramiento continuo en temas de progreso económico y social que procuren calidad de vida personal, familiar y social y que permitan crear conciencia de dignidad humana y posiciones positivas frente al envejecimiento.

\section{La educación para personas mayores}

Desde que se brinda un especial interés por los programas educativos de las personas de más edad, se ha acentuado la comprensión global de la educación de adultos, orientada no sólo hacia la actualización de los nuevos y más amplios conocimientos, sino, también, hacia la optimización de aquellos procesos que favorecen el propio desarrollo y la comunicación interpersonal, para lograr una efectiva integración en la sociedad.

Según varias teorías filosóficas, el ser humano nace "individuo" y, por la educación, llega a ser "persona”. La confianza, la aceptación del otro, el respeto, la acogida y la autenticidad son las nuevas actitudes de la relación educativa humanista que persigue, como objetivo educativo, su desarrollo armónico. Para conseguir que la persona realice todos los valores positivos con la debida jerarquía, surge la educación "integral" que ha de asegurar el perfeccionamiento de todos los aspectos de la personalidad: físicos, intelectuales, morales, afectivos, sociales, sexuales, estéticos, Quintana (1995). Desde una perspectiva más comprometida y trascendente, algunos autores lo llaman "educación para el desarrollo", para referirse a "la promoción y el estímulo de valores y actitudes dirigidas hacia la toma de conciencia, que lleve a emprender acciones justas en favor de la solidaridad entre los seres del planeta”.

Educar supone hacer dos trayectos, uno hacia sí mismo, otro hacia el entorno. Quien asume una verdadera educación acepta la transformación personal y la social. Las implicaciones educativas no pueden quedar aisladas. La educación de personas mayores, a pesar de que, a veces haya sido cuestionada, de ninguna forma carece de un valor social. Lejos del alcance de una comprensión positivista y mercantilista de la educación, también los mayores pueden ser "productivos".

\section{Educación permanente}

La educación permanente se considera como una corriente que pretende llevar la educación a todos los niveles y a todos los grupos generacionales y permitir eventos de enseñanza aprendizaje durante toda la vida.

En el contexto de la educación continua se manifiesta una de las grandes revoluciones que surgen en la época actual; se trata de llevar la educación a todos los niveles de la vida con la intención de que la reciban y la ejerciten todos y cada uno de los hombres. Es de gran importancia para el sujeto, el avance de su propia educación ya que las nuevas tecnologías son fuente de motivación 
Tdea Nora Hincapié Gil

y estímulo para el aprendizaje.

Los adultos jubilados no quieren permanecer como pasivos observadores de la vida, es su tiempo libre, y quieren ser partícipes de los acontecimientos que suceden en el entorno social, a través de la suscripción en los diferentes programas gerontológicos que les permite sentirse activos y útiles, para interactuar socialmente, adquirir nuevos conocimientos.

\section{Educación para adulto mayor: una propuesta hacia la inclusión social}

Desde que el hombre ha concebido la educación como una propuesta vinculante, producto de una reflexión constante, y que ha tenido como principio la construcción y elaboración del sujeto-individuo hacia la emancipación de todas sus facultades como humano. Es por eso que la educación, entendida como la manera en la que el hombre se piensa para desenvolver y potenciar sus facultades en lo personal y lo social, ha querido implementar estrategias, herramientas, arquetipos y dispositivos que apunten al enriquecimiento y fortalecimiento de las dimensiones del ser.

La educación debe ser en sí, una propuesta vinculante, donde se permita la elaboración, construcción y emancipación de la especie de lo humano por lo humano. No es gratuito que Paulo Freire (2004), mencione que en el proceso de dialogicidad en el acto educativo, la relación docente-alumno existe un vínculo inseparable en el acto de aprender, puesto que el que enseña aprende y el que aprende enseña. Una educación que promueva la inclusión y vinculación de todo su trasegar histórico en el consenso de la educación contemporánea. Una educación incluyente donde el docente y el estudiante sean partícipes de una construcción colectiva como aporte en la re-construcción de su experiencia en el mundo de la vida.

\section{Aspectos psicosociales}

Tener presente los factores que se presentan en la última etapa del ciclo vital, así como los cambios de tipo psicosocial como: las formas de convivencia, el grupo de apoyo que posean, y la etapa de jubilación, en lo cognitivo: las alteraciones de las funciones superiores, la atención, memoria, inteligencia, praxis y lenguaje $y$, con relación a lo afectivo; se presentan cambios de roles y las relaciones familiares y sociales se ven disminuidas por dificultades que se presentan en los adultos mayores, con los grupos generacionales.

Dentro de este contexto, el aprendizaje no puede concebirse como un proceso simple de asociación mecánica, pues en éste -el aprendizaje- influye la presencia de factores internos y externos que pueden alterar o afectar, de manera directa o indirecta, la forma de aprendizaje. Por ello, es necesario implementar estrategias participativas de carácter vivencial para llegar a la praxis de conocimientos adquiridos de acuerdo con las circunstancias y modificar su entorno.

\section{RESULTADOS}

- Diseño y divulgación de un plegable, poster $y$ video informativo con la fundamentación filosófica de la "Cátedra universitaria para adultos mayores”.

- El proyecto piloto fue admitido por el comité académico del IV Congreso iberoamericano de programas 
universitarios para adultos mayores en Alicante-España (2011).

- Demanda para inscripciones, matrículas y puesta en marcha del diplomado en "Educación permanente e inclusiva para adultos mayores".

- Ejecución de la prueba piloto de implementación con 40 adultos mayores y entrega de certificados en el diplomado "Educación permanente e inclusiva para adultos mayores" en el Tecnológico de Antioquia.

- Culminación del 100\% del diplomado, por los adultos mayores inscritos en el programa educativo.

- Participación activa de los adultos mayores en la construcción de la nueva propuesta denominada Cátedra universitaria para adultos mayores.

- Demanda para continuar el programa por extensión académica en el Tecnológico de Antioquia.

\section{DISCUSIÓN DE RESULTADOS}

Existen muchas experiencias mundiales de programas universitarios para adultos mayores dentro del campus universitario; como programas de extensión académica o educación continua, articulados a una de sus facultades, que buscan atender a la población adulta mayor en programas ocupacionales a través de la visión sociocultural e intergeneracional en procura de ofrecer una sociedad para todas las edades.

Los programas académicos para adultos mayores se conocen como: Programas Universitarios para Adultos Mayores, Aulas de la Experiencia, Universidades para la $3^{\text {a }}$ Edad, Universidades Abiertas, y algunas asociaciones como la Red Iberoamericana de Asociaciones de Adultos Mayores (Riaam), La Asociación Internacional de Universidades para la Tercera Edad y la Red de Universidades Abiertas, como referentes para las tendencias de una nueva modalidad educativa.

Como referente teórico en el transcurso de la investigación, se encuentran experiencias exitosas en países como España y Argentina, donde tienen una gran cobertura las universidades para adultos mayores, como programas de educación continua dentro del campus universitario y fortalecen los procesos académicos investigativos para generar alianzas de grupos de investigación de carácter multidisciplinar y transdisciplinar.

\section{CONCLUSIONES}

- Esta propuesta educativa permite, además del componente académico, participar en procesos de inclusión social, dentro del campus universitario, de acuerdo con las recomendaciones de las Naciones Unidas para el adulto mayor: "Hacia una sociedad para todas las edades".

- Los programas universitarios para adultos mayores, por sus objetivos y estructura organizativa, contribuyen enlacreación de una sociedad inclusiva, donde las acciones educativas muestran cómo la universidad, a la que asisten jóvenes y mayores de edades, constituye el marco idóneo paralas relaciones intergeneracionales. 
- Si la propuesta educativa se puede implementar en otras universidades, $y$ si tiene continuidad en el tiempo, se ha contribuido en la transformación social.

- La dimensión educativa, flexibilidad y abierta, establece cambios de buenas prácticas académicas, que permiten la visibilidad de los adultos mayores en contextos de inclusión social.

\section{Referencias}

Lemieux, André. (1997). Los programas universitarios para mayores. Madrid: Imserso.

Naciones Unidas (1982) Primera Asamblea Mundial sobre el Envejecimiento. Recuperado de:www.gerontologia.org/ portal/information/showInformation. php - Febrero de 2004.

Naciones Unidas (2002) Segunda Asamblea Mundial sobre el Envejecimiento. Internacional. Recuperado de www. imsersomayores.csic.es/internacional/ame/ index.html - Septiembre 2007

Freire, Paulo. (2004). Pedagogía de la Autonomía. Sao Paulo: Paz e Terra S.A. 